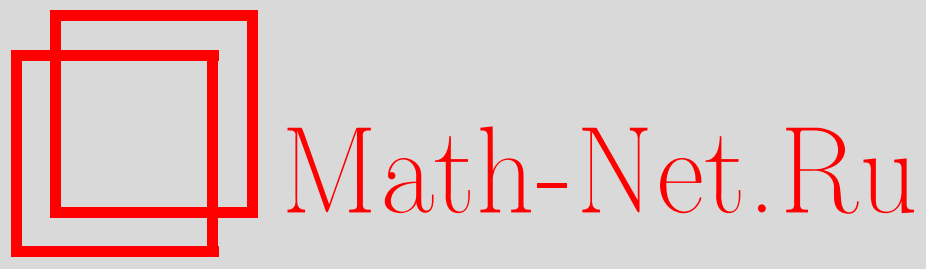

В. Ф. Молчанов, Канонические представления и надгруппы для гиперболоидов, Функи. анализ и его прил., 2005, том 39, выпуск 4, 48-61

DOI: https://doi.org/10.4213/faa84

Использование Общероссийского математического портала Math-Net.Ru подразумевает, что вы прочитали и согласны с пользовательским соглашением

http://www.mathnet.ru/rus/agreement

Параметры загрузки:

IP : 54.80 .97 .219

26 апреля 2023 г., 14:49:32

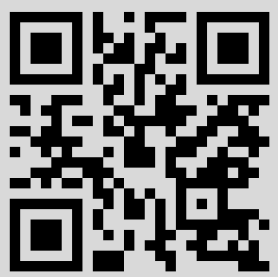


Функииональный анализ и его приложения

2005, т. 39, вып. 4, с. 48-61

УДК 517.98

\title{
Канонические представления и надгруппы для гиперболоидов*
}

\author{
(C) 2005. В. Ф. МолчАнов
}

Канонические представления на эрмитовых симметрических пространствах $G / K$ были введены Березиным [1] и Вершиком, Гельфандом и Граевым [2] для целей квантования и квантовой теории поля. Они унитарны относительно некоторого инвариантного нелокального скалярного произведения (форма Березина). Как мы можем сейчас видеть, естественно рассматривать канонические представления в более широком смысле: надо отказаться от условия унитарности и позволить представлениям действовать в более широких пространствах, в частности в пространствах обобщенных функций. Понятие канонического представления (в этом широком смысле) может быть распространено на другие классы полупростых симметрических пространств $G / H$, в частности на параэрмитовы симметрические пространства [7]. Более того, полезным оказывается рассматривать одновременно несколько пространств $G / H_{i}$, возможно с разными $H_{i}$, вложенными как открытые $G$-орбиты в некоторое компактное многообразие $\Omega$, на котором $G$ действует, так что $\Omega$ есть замыкание этих орбит.

Во всех этих случаях каноническое представление, обозначим его через $R$, может быть получено как ограничение на $G$ представления $\widetilde{R}$ (неприводимого, как правило) некоторой большей группы («надгруппы») $\widetilde{G}$.

В этой работе мы рассматриваем псевдоортогональную группу $G=\mathrm{SO}_{0}(p, q)$ и гиперболоид $\mathscr{X}=G / H$, где $H=\mathrm{SO}_{0}(p, q-1)$. В качестве надгруппы мы берем псевдоортогональную группу $\widetilde{G}=\mathrm{SO}_{0}(p+1, q)$. Мы определяем канонические представления $R_{\lambda, \nu}, \lambda \in \mathbb{C}, \nu=0,1$, как ограничения на $G$ представлений $\widetilde{R}_{\lambda, \nu}$ группы $\widetilde{G}$, связанных с конусом, см. [3]. Они действуют на компактном многообразии $\Omega$, которое является прямым произведением двух сфер размерностей $p$ и $q-1$. Это многообразие $\Omega$ содержит два экземпляра пространства $\mathscr{X}$ и является их замыканием. Канонические пространства порождают граничные представления группы $G$, связанные с границей обеих $G$-орбит $\mathscr{X}$ на многообразии $\Omega$. Цель настоящей работы состоит в том, чтобы выяснить взаимодействие операторов Ли группы $\widetilde{G}$ в $\widetilde{R}_{\lambda, \nu}$ (т. е. взаимодействие представления $\widetilde{R}_{\lambda, \nu}$ алгебры Ли $\widetilde{\mathfrak{g}}$ группы $\widetilde{G}$ ) с операторами, сплетающими канонические представления $R_{\lambda, \nu}$ и представления группы $G$, связанные с конусом (мы назовем эти операторы преобразованиями Пуассона и преобразованиями Фурье, связанными с каноническими представлениями). Это развивает идею Неретина [12] в модифицированной форме. Такая форма была предложена в работе

* Работа поддержана грантами РФФИ 01-01-0100-а, Минобразования РФ Е02-1.0-156, Научной программы «Университеты России» ур.04.01.037 и NWO (Голландской организации научных исследований) 047-008-009. 
автора [9], см. также [10]. С другой стороны, эту задачу можно рассматривать как некоторую версию классической задачи о действии группы (алгебры Ли) в базисе, собственном для некоторой подгруппы. Многообещающей является также связь этой задачи с полиномиальным квантованием на параэрмитовых пространствах, см., например, [8].

Интересно отметить, что формулы взаимодействия для элементов из алгебры Ли $\widetilde{\mathfrak{g}}$, не входящих в алгебру Ли $\mathfrak{g}$, содержат дифференциальные операторы второго порядка.

В основной части статьи мы рассматриваем общий случай: $p>1, q>1$. В последнем параграфе мы указываем отличия случаев $p=1$ или $q=1$, см. [9-11], от общего случая. Эти случаи оказываются более простыми.

Мы не касаемся здесь задачи разложения канонических и граничных представлений, это будет сделано в другой работе.

Приведем сначала результат для исходного случая - для канонических представлений из [2]. Ограничимся преобразованием Пуассона. Представление $\widetilde{R}_{\lambda}$, $\lambda \in \mathbb{C}$, надгруппы $\widetilde{G}=\mathrm{SL}(2, \mathbb{C})$ (двукратного накрытия группы $\left.\mathrm{SO}_{0}(3,1)\right)$ действует в функциях $f(z)$ комплексного переменного $z$ по формуле

$$
\left(\widetilde{R}_{\lambda}(g) f\right)(z)=f\left(\frac{\alpha z+\gamma}{\beta z+\delta}\right)|\beta z+\delta|^{-2 \lambda-4}, \quad g=\left(\begin{array}{ll}
\alpha & \beta \\
\gamma & \delta
\end{array}\right) .
$$

Каноническое представление $R_{\lambda}$ группы $G=\mathrm{SU}(1,1)$ - это ограничение представления $\widetilde{R}_{\lambda}$ на $G$ и на функции в единичном круге $D=\{z \in \mathbb{C}: z \bar{z}<1\}$. Возьмем какой-нибудь базис в алгебре Ли $\mathfrak{g}$ группы $G$ и дополним его до базиса алгебры Ли $\widetilde{\mathfrak{g}}$ группы $\widetilde{G}$ матрицами

$$
M_{1}=\left(\begin{array}{cc}
0 & 1 / 2 \\
-1 / 2 & 0
\end{array}\right), \quad M_{2}=\left(\begin{array}{cc}
0 & i / 2 \\
i / 2 & 0
\end{array}\right), \quad M_{3}=\left(\begin{array}{cc}
-1 / 2 & 0 \\
0 & 1 / 2
\end{array}\right) .
$$

Элементарные представления $T_{\sigma}, \sigma \in \mathbb{C}$, группы $G$ действуют в пространстве $\mathscr{D}(S)$ (обозначение $\mathscr{D}(M)$ см. ниже) на единичной окружности $S=\left\{s: s=e^{i t}\right.$, $t \in \mathbb{R}\}$ по формуле

$$
\left(T_{\sigma}(g) \varphi\right)(s)=\varphi\left(\frac{a s+\bar{b}}{b s+\bar{a}}\right)|b s+\bar{a}|^{2 \sigma}, \quad g=\left(\begin{array}{ll}
a & b \\
\bar{b} & \bar{a}
\end{array}\right) .
$$

Преобразование Пуассона $P_{\lambda, \sigma}$ задается формулой

$$
\left(P_{\lambda, \sigma} \varphi\right)(z)=(1-z \bar{z})^{-\lambda-\sigma-2} \int_{S}|1-z \bar{s}|^{2 \sigma} \varphi(s) d s \quad(d s=d t) .
$$

Оно сплетает $T_{-\sigma-1}$ и $R_{\lambda}$, так что

$$
R_{\lambda}(X) P_{\lambda, \sigma}=P_{\lambda, \sigma} T_{-\sigma-1}(X), \quad X \in \mathfrak{g} .
$$

Для $M_{k}, k=1,2,3$, имеем

$$
\widetilde{R}_{\lambda}\left(M_{k}\right) P_{\lambda, \sigma}=a(\lambda, \sigma) P_{\lambda, \sigma+1} \circ K_{\sigma}^{(k)}+b(\lambda, \sigma) P_{\lambda, \sigma-1} \circ C^{(k)},
$$


где $C^{(1)}, C^{(2)}, C^{(3)}$ - умножение на $-\cos t, \sin t,-1$ соответственно,

$$
\begin{gathered}
a(\lambda, \sigma)=\frac{\lambda+\sigma+2}{(\sigma+1)(2 \sigma+1)}, \quad b(\lambda, \sigma)=\frac{\sigma(\lambda-\sigma+1)}{2 \sigma+1}, \\
K_{\sigma}^{(1)} \varphi=-\cos t \cdot \varphi^{\prime \prime}+(2 \sigma+3) \sin t \cdot \varphi^{\prime}+(\sigma+1)(\sigma+2) \cos t \cdot \varphi, \\
K_{\sigma}^{(2)} \varphi=\sin t \cdot \varphi^{\prime \prime}+(2 \sigma+3) \cos t \cdot \varphi^{\prime}-(\sigma+1)(\sigma+2) \sin t \cdot \varphi, \\
K_{\sigma}^{(3)} \varphi=-\varphi^{\prime \prime}-(\sigma+1)^{2} \varphi,
\end{gathered}
$$

а штрих обозначает дифференцирование по $t$. См. также конец $\S 6$.

Введем некоторые обозначения и соглашения.

Мы используем следующее обозначение для характера группы $\mathbb{R}^{*}=\mathbb{R} \backslash\{0\}$ :

$$
t^{\lambda, m}=|t|^{\lambda}(\operatorname{sgn} t)^{m}
$$

где $t \in \mathbb{R}^{*}, \lambda \in \mathbb{C}, m \in \mathbb{Z}$. Этот характер зависит от $m$ по модулю 2 . Пусть $\mathbb{N}=\{0,1,2, \ldots\}$.

Если $M$ - многообразие, то $\mathscr{D}(M)$ обозначает пространство Шварца бесконечно дифференцируемых функций на $M$ с комплексными значениями и компактным носителем, снабженное обычной топологией, а $\mathscr{D}^{\prime}(M)$ обозначает пространство обобщенных функций на $M$ - линейных непрерывных функционалов на $\mathscr{D}(M)$.

Если представление группы Ли дифференцируемо, то мы используем один и тот же символ для него и для соответствующего представления алгебры Ли этой группы Ли.

Пусть $(\cdot, \cdot)$ - билинейная невырожденная форма на $\mathscr{D}(M)$, инвариантная относительно пары представлений $(T, S)$ группы $G$, действующих на $M$ :

$$
(T(g) F, f)=\left(F, S\left(g^{-1}\right) f\right) .
$$

Тогда мы можем распространить представление $T$ на $\mathscr{D}^{\prime}(M)$ посредством формулы $(0.3)$, где $(F, f)$ обозначает значение обобщенной функции $F$ на функции $f \in \mathscr{D}(M)$. Это на самом деле расширение, поскольку $\mathscr{D}(M)$ вкладывается в $\mathscr{D}^{\prime}(M)$, если мы сопоставляем функции $f \in \mathscr{D}(M)$ функционал $h \mapsto(f, h)$ с помощью формы $(\cdot, \cdot)$. Мы сохраняем символ (в данном случае $T$ ) для этого расширения.

\section{§1. Псевдоортогональная группа и гиперболоид}

Пусть $G$ есть группа $\mathrm{SO}_{0}(p, q)$ - связная компонента единицы в группе линейных преобразований пространства $\mathbb{R}^{n}, n=p+q$, сохраняющих билинейную форму

$$
[x, y]=-x_{1} y_{1}-\cdots-x_{p} y_{p}+x_{p+1} y_{p+1}+\cdots+x_{n} y_{n} .
$$

Мы будем считать, что $G$ действует в $\mathbb{R}^{n}$ справа, и в соответствии с этим считаем, что векторы - это строки.

Пусть $K$ - подгруппа в $G$, состоящая из элементов $g$, таких, что $g=I g I$, где $I=\operatorname{diag}\left\{\delta_{1}, \ldots, \delta_{n}\right\}$,

$$
\delta_{1}=\cdots=\delta_{p}=-1, \quad \delta_{p+1}=\cdots=\delta_{n}=1 .
$$

Это - максимальная компактная подгруппа в $G$, она изоморфна $\mathrm{SO}(p) \times \mathrm{SO}(q)$. 
Алгебра Ли $\mathfrak{g}$ группы $G$ состоит из вещественных матриц $X$ порядка $n$, удовлетворяющих соотношению $X^{\prime}=-I X I$, где штрих означает транспонирование. Базис в $\mathfrak{g}$ образован матрицами $L_{i j}=E_{i j}-\delta_{i} \delta_{j} E_{j i}, i<j$, где $E_{i j}-$ «матричная единица» (1 на месте $(i, j)$ и 0 на остальных местах).

Алгебра Ли $\mathfrak{g}$ распадается в прямую сумму $\mathfrak{k}+\mathfrak{p}$, где $\mathfrak{k}$ - алгебра Ли группы $K$, a $\mathfrak{p}$ - подпространство, ортогональное $\mathfrak{k}$ относительно формы Киллинга. Базис в $\mathfrak{k}$ образован матрицами $L_{i j}$ с $i<j \leqslant p$ и $p<i<j$, базис в $\mathfrak{p}$ образован матрицами $L_{i j}, i \leqslant p<j$.

Пусть $\mathscr{X}$ обозначает гиперболоид $\{x:[x, x]=1\}$. Это - однородное пространство группы $G$ относительно сдвигов $x \mapsto x g$. Стационарная подгруппа $H$ точки $x^{0}=(0, \ldots, 0,1)$ есть $\operatorname{SO}_{0}(p, q-1)$. Мера на $\mathscr{X}$, инвариантная относительно $G$, в координатах $x_{1}, \ldots, \widehat{x}_{k}, \ldots, x_{n}$ (переменная $x_{k}$ должна быть опущена) - это

$$
d x=\left|x_{k}\right|^{-1} d x_{1} \cdots \widehat{d x_{k}} \cdots d x_{n}
$$

(разные $k$ дают одну и ту же меру).

Пусть $U$ - представление группы $G$ в функциях на $\mathscr{X}$ сдвигами: $(U(g) f)(x)$ $=f(x g)$. Пространство, в котором действует $U$, будет специально указываться, если нужно.

\section{§2. Представления группы $G$, связанные с конусом}

Напомним [3] некоторый материал о представлениях группы $G$, связанных с конусом. Мы используем «компактную картину». В основной части текста - в $\S \S 2-5$ - мы рассматриваем общий случай $p>1, q>1$.

Для $x \in \mathbb{R}^{n}$ положим $|x|=\left(x_{1}^{2}+\cdots+x_{p}^{2}\right)^{1 / 2}$. Пусть $S$ - сечение конуса $\{x:[x, x]=0\}$, состоящее из точек $s$, таких, что $|s|=1$. Оно есть прямое произведение двух единичных сфер $S_{1} \subset \mathbb{R}^{p}$ и $S_{2} \subset \mathbb{R}^{q}$, задаваемых уравнениями $s_{1}^{2}+\cdots+s_{2}^{2}=1$ и $s_{p+1}^{2}+\cdots+s_{n}^{2}=1$ соответственно. Пусть $d s-$ евклидова мера на $S$. В качестве локальных координат на $S_{1}$ и $S_{2}$ возьмем соответственно переменные $s_{i}, 1 \leqslant i \leqslant p$, и $s_{j}, p<j \leqslant n$, опуская по одной из них для каждой из сфер. Операторы Лапласа-Бельтрами $\Delta_{1}$ и $\Delta_{2}$ на $S_{1}$ и $S_{2}$ задаются формулами

$$
\Delta_{1}=H_{1}-D_{1}^{2}-(p-2) D_{1}, \quad \Delta_{2}=H_{2}-D_{2}^{2}-(q-2) D_{2},
$$

где

$$
H_{1}=\sum \frac{\partial^{2}}{\partial s_{i}^{2}}, \quad D_{1}=\sum s_{i} \frac{\partial}{\partial s_{i}}, \quad H_{2}=\sum \frac{\partial^{2}}{\partial s_{j}^{2}}, \quad D_{2}=\sum s_{j} \frac{\partial}{\partial s_{j}} .
$$

Нам потребуется разность этих операторов

$$
\Delta^{(-)}=-\Delta_{1}+\Delta_{2}
$$

Пусть $\sigma \in \mathbb{C}, \varepsilon=0,1$. Обозначим через $\mathscr{D}_{\varepsilon}(S)$ пространство функций $\varphi$ из $\mathscr{D}(S)$ четности $\varepsilon: \varphi(-s)=(-1)^{\varepsilon} \varphi(s)$. Представление $T_{\sigma, \varepsilon}$ группы $G$ действует в $\mathscr{D}_{\varepsilon}(S)$ так:

$$
\left(T_{\sigma, \varepsilon}(g) \varphi\right)(s)=\varphi(s g /|s g|) \cdot|s g|^{\sigma} .
$$

Если $\sigma$ не целое, то $T_{\sigma, \varepsilon}$ неприводимо и эквивалентно представлению $T_{2-n-\sigma, \varepsilon}$. Билинейная форма

$$
(\psi, \varphi)_{S}=\int_{S} \psi(s) \varphi(s) d s
$$


инвариантна относительно пары $\left(T_{\sigma, \varepsilon}, T_{2-n-\sigma, \varepsilon}\right)$, т. е.

$$
\left(T_{\sigma, \varepsilon}(g) \psi, \varphi\right)_{S}=\left(\psi, T_{2-n-\sigma, \varepsilon}\left(g^{-1}\right) \varphi\right)_{S}
$$

Для $X \in \mathfrak{g}$ это дает

$$
\left(T_{\sigma, \varepsilon}(X) \psi, \varphi\right)_{S}=-\left(\psi, T_{2-n-\sigma, \varepsilon}(X) \varphi\right)_{S}
$$

С помощью формулы (2.4) представление $T_{\sigma, \varepsilon}$ может быть распространено на пространство $\mathscr{D}_{\varepsilon}^{\prime}(S)$ обобщенных функций на $S$ четности $\varepsilon$. В обозначениях иногда удобно вместо $\varepsilon$ писать другое целое число той же четности.

Выпишем операторы, отвечающие в представлении $T_{\sigma, \varepsilon}$ элементам базиса $L_{i j}$ (эти операторы не зависят от $\varepsilon$, поэтому $\varepsilon$ мы опустим):

$$
\begin{array}{llrl}
T_{\sigma}\left(L_{i k}\right) & =-s_{k} \frac{\partial}{\partial s_{i}}+s_{i} \frac{\partial}{\partial s_{k}}, & & L_{i k} \in \mathfrak{k}, \\
T_{\sigma}\left(L_{i k}\right) & =s_{k} X_{i}+s_{i} Y_{k}+\sigma s_{i} s_{k}, & & L_{i k} \in \mathfrak{p},
\end{array}
$$

где

$$
X_{i}=\frac{\partial}{\partial s_{i}}-s_{i} D_{1}, \quad Y_{k}=\frac{\partial}{\partial s_{k}}-s_{k} D_{2} .
$$

Если какая-нибудь переменная $s_{m}$ не входит в число локальных координат, то производную по ней надо опустить. Обратно, операторы $X_{i}, Y_{k}(i \leqslant p<k)$ выражаются через операторы Ли:

$$
\begin{aligned}
& \sum_{k} s_{k} T_{\sigma}\left(L_{i k}\right)=X_{i}+\sigma s_{i}, \\
& \sum_{i} s_{i} T_{\sigma}\left(L_{i k}\right)=Y_{k}+\sigma s_{k} .
\end{aligned}
$$

Выпишем операторы, сопряженные операторам $X_{i}, Y_{k}$ относительно формы $(2.3)$ :

$$
X_{i}^{*}=-X_{i}+(p-1) s_{i}, \quad Y_{k}^{*}=-Y_{k}+(q-1) s_{k} .
$$

Для получения этих формул проще всего использовать формулы (2.9), (2.10), затем (2.5) и затем снова $(2.9),(2.10)$. Нам еще потребуется оператор, сопряженный оператору $s_{i} \Delta^{(-)}$, т. е. оператор $\Delta^{(-)} \circ s_{i}$. Имеем

$$
\begin{aligned}
\left(s_{i} \Delta^{(-)}\right)^{*}=s_{i} \Delta^{(-)}-2 X_{i}+(p-1) s_{i}, & & i \leqslant p, \\
\left(s_{k} \Delta^{(-)}\right)^{*}=s_{k} \Delta^{(-)}+2 Y_{k}-(q-1) s_{k}, & & k>p .
\end{aligned}
$$

\section{§3. Канонические представления на гиперболоиде}

Расширим пространство $\mathbb{R}^{n}$, где действует $G$, до пространства $\mathbb{R}^{n+1}$, добавив координату $x_{0}$, и снабдим $\mathbb{R}^{n+1}$ билинейной формой

$$
[[x, y]]=-x_{0} y_{0}-x_{1} y_{1}-\cdots-x_{p} y_{p}+x_{p+1} y_{p+1}+\cdots+x_{n} y_{n}
$$

Группа $\widetilde{G}=\mathrm{SO}_{0}(p+1, q)$ сохраняет эту форму. Как и раньше, считаем, что $\widetilde{G}$ действует на $\mathbb{R}^{n+1}$ справа. Пусть $\widetilde{\mathfrak{g}}$ - алгебра Ли группы $\widetilde{G}$.

Напомним еще раз некоторые факты о представлениях группы $\widetilde{G}$, связанных с конусом [3]. Обозначения мы адаптируем к нашим целям. 
Пусть $\mathscr{C}$ обозначает конус $\{x:[[x, x]]=0, x \neq 0\}$ в $\mathbb{R}^{n+1}$. Пусть $\mathscr{D} \lambda, \nu(\mathscr{C})$, где $\lambda \in \mathbb{C}, \nu=0,1$, обозначает пространство функций класса $C^{\infty}$ на $\mathscr{C}$ (с комплексными значениями), однородных степени $\lambda, \nu$, т. е.

$$
f(t x)=t^{\lambda, \nu} f(x), \quad x \in \mathscr{C}, t \in \mathbb{R}^{*} .
$$

Представление $\widetilde{R}_{\lambda, \nu}$ группы $\widetilde{G}$ действует сдвигами в $\mathscr{D}_{1-n-\lambda, \nu}$ (из некоторых соображений, впрочем, не принципиальных, мы берем здесь $1-n-\lambda$, а не $\lambda$ ):

$$
\left(\widetilde{R}_{\lambda, \nu}(g) f\right)(x)=f(x g), \quad x \in \mathscr{C}, g \in \widetilde{G} .
$$

Для $x \in \mathbb{R}^{n+1}$ положим $\|x\|=\left(x_{0}^{2}+x_{1}^{2}+\cdots+x_{p}^{2}\right)^{1 / 2}$. Пусть $\Omega-$ множество точек $u$ из $\mathscr{C}$, таких, что $\|u\|=1$. Это прямое произведение единичных сфер из $\mathbb{R}^{p+1}$ и $\mathbb{R}^{q}$. Пусть $d u$ - евклидова мера на $\Omega$.

Функции из $\mathscr{D}_{\lambda, \nu}(\mathscr{C})$ полностью определяются своими ограничениями на $\Omega$. Эти ограничения образуют пространство $\mathscr{D}_{\nu}(\Omega)$ функций из $\mathscr{D}(\Omega)$ четности $\nu$. Представление $\widetilde{R}_{\lambda, \nu}$ реализуется на $\mathscr{D}_{\nu}(\Omega)$ так:

$$
\left(\widetilde{R}_{\lambda, \nu}(g) f\right)(u)=f(u g /\|u g\|) \cdot\|u g\|^{1-n-\lambda} .
$$

Билинейная форма

$$
(f, h)_{\Omega}=\int_{\Omega} f(u) h(u) d u
$$

инвариантна относительно пары $\left(\widetilde{R}_{\lambda, \nu}, \widetilde{R}_{1-n-\lambda, \nu}\right)$ :

$$
\left(\widetilde{R}_{\lambda, \nu}(g) f, h\right)_{\Omega}=\left(f, \widetilde{R}_{1-n-\lambda, \nu}\left(g^{-1}\right) h\right)_{\Omega} .
$$

Формула (3.4) позволяет распространить $\widetilde{R}_{\lambda, \nu}$ на пространство $\mathscr{D}_{\nu}^{\prime}(\Omega)$ обобщенных функций на $\Omega$ четности $\nu$.

Вложим группу $G$ в группу $\widetilde{G}$ как подгруппу, сохраняющую координату $x_{0}$. Каноническим представлением $R_{\lambda, \nu}, \lambda \in \mathbb{C}, \nu=0,1$, группы $G$ назовем ограничение на $G$ представления $\widetilde{R}_{\lambda, \nu}$. Оно действует на $\mathscr{D}_{\nu}(\Omega)$, а также распространяется на $\mathscr{D}_{\nu}^{\prime}(\Omega)$.

Рассмотрим сечение $\widetilde{\mathscr{X}}$ конуса $\mathscr{C}$ гиперплоскостью $x_{0}=1$. Мы можем отождествить его с гиперболоидом $\mathscr{X}$ : точке $x=\left(x_{1}, \ldots, x_{n}\right)$ из $\mathscr{X}$ сопоставляем точку $\widetilde{x}=\left(1, x_{1}, \ldots, x_{n}\right)$ из $\widetilde{\mathscr{X}}$. Ограничим функции из $\mathscr{D}_{1-n-\lambda, \nu}(\mathscr{C})$ на $\widetilde{\mathscr{X}}=\mathscr{X}$. Мы получим некоторое пространство $\mathscr{D}_{1-n-\lambda, \nu}(\mathscr{X})$ функций на $\mathscr{X}$. Оно содержит $\mathscr{D}(\mathscr{X})$ и содержится в $C^{\infty}(\mathscr{X})$. На этом пространстве (а также на $\mathscr{D}(\mathscr{X}))$ представление $R_{\lambda, \nu}$ становится представлением $U$ сдвигами.

На пространстве $\mathscr{D}_{\nu}(\Omega)$ представление $R_{\lambda, \nu}$ действует по формуле $(3.2)$ с $g \in G$. Билинейная форма (3.3) инвариантна относительно пары $\left(R_{\lambda, \nu}, R_{1-n-\lambda, \nu}\right)$.

Группа $G$ имеет три орбиты на $\Omega$ относительно действия $u \mapsto u g /|u g|$, а именно, две орбиты $\Omega^{+}=\left\{u: u_{0}>0\right\}$ и $\Omega^{-}=\left\{u: u_{0}<0\right\}$ размерности $n-1$ и одну орбиту $\Omega_{0}=\left\{u: u_{0}=0\right\}$ размерности $n-2$. Последняя может быть отождествлена с многообразием $S$ из $\S 2$ : точке $s=\left(s_{1}, \ldots, s_{n}\right)$ из $S$ сопоставляем точку $\stackrel{\circ}{s}=\left(0, s_{1}, \ldots, s_{n}\right)$ из $\Omega_{0}$. Положим $\Omega^{\prime}=\Omega^{+} \cup \Omega^{-}$. Каждая из орбит $\Omega^{ \pm}$может быть отождествлена с сечением $\widetilde{\mathscr{X}}$ и, следовательно, с гиперболоидом $\mathscr{X}$. Отображение строится по прямолинейным образующим конуса: точке 
$u$ из $\Omega$ сопоставляется точка $\widetilde{x}=u / u_{0}$ из $\widetilde{\mathscr{X}}$. Меры на $\mathscr{X}$ и на $\Omega$ связаны так:

$$
d x=\left|u_{0}\right|^{1-n} d u \text {. }
$$

Пусть функция $f$ принадлежит $\mathscr{D}_{1-n-\lambda, \nu}(\mathscr{C})$. Ее ограничения $f(u)$ на $\Omega$ и $f(x)$ на $\widetilde{\mathscr{X}}=\mathscr{X}$ связаны так:

$$
f(u)=u_{0}^{1-n-\lambda, \nu} f(x), \quad x \longleftrightarrow \widetilde{x}=u / u_{0} .
$$

Каноническое представление $R_{\lambda, \nu}$ порождает следующие представления $L_{\lambda, \nu}$ и $M_{\lambda, \nu}$, связанные с границей $\Omega_{0}$ многообразий $\Omega^{ \pm}$(граничные представления).

Пусть $f$ - функция из $\mathscr{D}_{\nu}(\Omega)$. Рассмотрим ее ряд Тейлора $a_{0}+a_{1} u_{0}+$ $a_{2} u_{0}^{2}+\cdots$ по степеням $u_{0}$. Здесь $a_{m}=a_{m}(f)-$ функции из $\mathscr{D}_{\nu-m}(S)$. Определим отображение $u \mapsto s(u)$ точек $u$ из $\Omega$ с $u_{0} \neq \pm 1$ на $S$ следующим образом: $s_{i}(u)=\left(1-u_{0}^{2}\right)^{-1 / 2} u_{i}$ для $i=1, \ldots, p$ и $s_{i}(u)=u_{i}$ для $i=p+1, \ldots, n$. Обозначим через $\Sigma_{\nu}^{k}(\Omega), k \in \mathbb{N}, \nu=0,1$, пространство обобщенных функций из $\mathscr{D}_{\nu}^{\prime}(\Omega)$, имеющих вид

$$
\sum_{m=0}^{k} \varphi_{m}(s(u)) \delta^{(m)}\left(u_{0}\right),
$$

где $\varphi_{m} \in \mathscr{D}_{\nu-m}(S), \delta$ - дельта-функция Дирака на прямой и $\delta^{(m)}-$ ее производные. Пусть $\Sigma_{\nu}(\Omega)=\bigcup \Sigma_{\nu}^{k}(\Omega)$.

Обозначим через $L_{\lambda, \nu}$ ограничение представления $R_{\lambda, \nu}$ на $\Sigma_{\nu}(\Omega)$. Это представление записывается верхней треугольной матрицей с диагональю $T_{2-n-\lambda+m, \nu-m}, m \in \mathbb{N}$. Для $\lambda$ общего положения (в частности, $2 \lambda \notin \mathbb{Z}$ ) представление $L_{\lambda, \nu}$ диагонализуемо (диагональ та же).

Пусть $a(f)$ обозначает столбец из коэффициентов Тейлора $a_{m}(f)$. Представление $M_{\lambda, \nu}$ действует на этих столбцах:

$$
M_{\lambda, \nu}(g) a(f)=a\left(R_{\lambda, \nu}(f)\right) .
$$

Оно записывается нижней треугольной матрицей с диагональю $T_{1-n-\lambda-m, \nu-m}$, $m \in \mathbb{N}$. Для $\lambda$ общего положения оно диагонализуемо.

\section{§4. Преобразования Пуассона и Фурье}

Пространство $H$-инвариантов в $\mathscr{D}_{\varepsilon}^{\prime}(S)$ относительно $T_{\sigma, \varepsilon}$ одномерно, базисом служит обобщенная функция

$$
\theta_{\sigma, \varepsilon}(s)=s_{n}^{\sigma, \varepsilon}=\left[x^{0}, s\right]^{\sigma, \varepsilon}
$$

или ее вычеты в полюсах по $\sigma$ (в точках $\sigma \in-1-\varepsilon-2 \mathbb{N}$ ), см. [4]. Согласно общей схеме [6], этот $H$-инвариант порождает ядро Пуассона

$$
P_{\sigma, \varepsilon}(x, s)=\left(T_{\sigma, \varepsilon}\left(g^{-1}\right) \theta_{\sigma, \varepsilon}\right)(s)=[x, s]^{\sigma, \varepsilon},
$$

где $x=x^{0} g \in \mathscr{X}, s \in S$. Это ядро порождает два оператора: преобразование

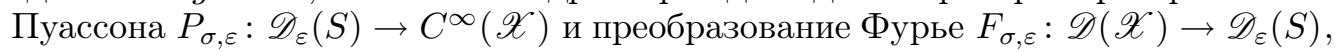
задаваемые формулами

$$
\begin{aligned}
\left(P_{\sigma, \varepsilon} \varphi\right)(x) & =\int_{S}[x, s]^{\sigma, \varepsilon} \varphi(s) d s \\
\left(F_{\sigma, \varepsilon} f\right)(s) & =\int_{\mathscr{X}}[x, s]^{\sigma, \varepsilon} f(x) d x,
\end{aligned}
$$


Интегралы абсолютно сходятся при $\operatorname{Re} \sigma>-1$ и продолжаются аналитически по $\sigma$ до мероморфных функций (с полюсами в тех же точках, что и у $\theta_{\sigma, \varepsilon}$ ). Оба преобразования - линейные непрерывные операторы, они сплетают $T_{2-n-\sigma, \varepsilon}$ с $U$ и $U$ с $T_{\sigma, \varepsilon}$ соответственно.

Отметим, что для $\sigma$ общего положения образ преобразования Пуассона плотен в пространстве функций из $C^{\infty}(\mathscr{X})$ четности $\varepsilon$, собственных для оператора Лапласа-Бельтрами на $\mathscr{X}$ с собственным значением $\sigma(\sigma+n-2)$. Всякая функция из $\mathscr{D}(\mathscr{X})$ восстанавливается по ее компонентам Фурье непрерывной серии $(\operatorname{Re} \sigma=(2-n) / 2)$ и первой дискретной серии (по поводу этих серий см. [3]), и имеет место формула Планшереля (см. [5]).

Теперь построим операторы, сплетающие канонические представления $R_{\lambda, \nu}$ и представления $T_{\sigma, \varepsilon}$.

Для функции $\varphi \in \mathscr{D}_{\varepsilon}(S)$ рассмотрим ее преобразование Пуассона $P_{\sigma, \varepsilon} \varphi$ как ограничение на $\widetilde{\mathscr{X}}=\mathscr{X}$ некоторой функции на конусе $\mathscr{C}$ степени однородности $(1-n-\lambda, \nu)$, см. (3.1), и затем перейдем от $\widetilde{\mathscr{X}}$ к $\Omega$. Для этого в формуле $(4.2)$ мы переписываем $[x, s]$ как $[[\widetilde{x}, \stackrel{\circ}{s}]]$ (см. $\S 3$ по поводу $\widetilde{x}$ и $\stackrel{\circ}{s})$ и затем используем соотношение (3.6). Мы получим оператор $P_{\lambda, \nu ; \sigma, \varepsilon}: \mathscr{D}_{\varepsilon}(S) \rightarrow C^{\infty}\left(\Omega^{\prime}\right)$, задаваемый формулой

$$
\left(P_{\lambda, \nu ; \sigma, \varepsilon} \varphi\right)(u)=u_{0}^{1-n-\lambda-\sigma, \nu-\varepsilon} \int_{S}[[u, \stackrel{\circ}{s}]]^{\sigma, \varepsilon} \varphi(s) d s
$$

(мы используем здесь обозначение (0.2); напомним еще, что для $s \in S$ имеем $\left.[[u, \stackrel{\circ}{s}]]=-u_{1} s_{1}-\cdots-u_{p} s_{p}+u_{p+1} s_{p+1}+\cdots+u_{n} s_{n}\right)$. Этот оператор сплетает $T_{2-n-\sigma, \varepsilon}$ с $R_{\lambda, \nu}$. Назовем его преобразованием Пуассона, связанным с каноническим представлением $R_{\lambda, \nu}$. Здесь мы рассматриваем $R_{\lambda, \nu}$ как ограничение на $C_{\nu}^{\infty}\left(\Omega^{\prime}\right)$ (пространство функций из $C^{\infty}\left(\Omega^{\prime}\right)$ четности $\nu$ ) представления $R_{\lambda, \nu}$, действующего в пространстве $\mathscr{D}_{\nu}^{\prime}(\Omega)$.

Для $K$-финитных функций $\varphi$ и для $\sigma$ общего положения (в частности, если $2 \sigma \notin \mathbb{Z})$ это преобразование Пуассона может быть разложено по степеням $u_{0}$ :

$$
\begin{gathered}
\left(P_{\lambda, \nu ; \sigma, \varepsilon} \varphi\right)(u)=u_{0}^{1-n-\lambda-\sigma, \nu-\varepsilon} \sum_{k=0}^{\infty}\left(C_{\sigma, \varepsilon, k} \varphi\right)(s(u)) u_{0}^{2 k} \\
\quad+u_{0}^{-\lambda+\sigma-1, \nu-\varepsilon} \sum_{k=0}^{\infty}\left(\mathscr{D}_{\sigma, \varepsilon, k} \varphi\right)(s(u)) u_{0}^{2 k},
\end{gathered}
$$

где $C_{\sigma, \varepsilon, k}, \mathscr{D}_{\sigma, \varepsilon, k}-$ некоторые линейные непрерывные операторы в $\mathscr{D}_{\varepsilon}(S)$. Множители перед рядами в (4.5) дают полюсы преобразования Пуассона по $\sigma$, зависящие от $\lambda$; они располагаются в точках

$$
\sigma=\lambda-k, \quad \sigma=2-n-\lambda+l,
$$

где $k, l \in \mathbb{N}$ и $k \equiv \nu-\varepsilon, l \equiv \nu-\varepsilon(\bmod 2)$.

Аналогично, рассмотрим функцию $f$ в формуле (4.3) как ограничение на $\widetilde{\mathscr{X}}=\mathscr{X}$ некоторой функции из $\mathscr{D}_{1-n-\lambda, \nu}(\mathscr{C})$, затем переписываем $[x, s]$ как $[[\widetilde{x}, \stackrel{\circ}{s}]]$ и используем формулы (3.6), (3.5). Мы получим интеграл по $\Omega$ с множителем $1 / 2$, поскольку отображение $u \mapsto \widetilde{x}=x$ накрывает $\mathscr{X}$ дважды. Отбрасывая этот множитель, мы получим оператор $F_{\lambda, \nu ; \sigma, \varepsilon}: \mathscr{D}_{\nu}(\Omega) \rightarrow \mathscr{D}_{\varepsilon}(S)$, задаваемый 
формулой

$$
\left(F_{\lambda, \nu ; \sigma, \varepsilon} f\right)(s)=\int_{\Omega}[[u, \stackrel{\circ}{s}]]^{\sigma, \varepsilon} u_{0}^{\lambda-\sigma, \nu-\varepsilon} f(u) d u .
$$

Интеграл абсолютно сходится при $\operatorname{Re} \sigma>-1 / 2, \operatorname{Re}(\lambda \pm \sigma)>-1$ и аналитически продолжается по $\sigma$ и $\lambda$ до мероморфной функции. Назовем оператор $F_{\lambda, \nu ; \sigma, \varepsilon}$ преобразованием Фуръе, связанным с каноническим преобразованием $R_{\lambda, \nu}$. $\mathrm{O}_{\mathrm{H}}$ сплетает $R_{\lambda, \nu}$ с $T_{\sigma, \varepsilon}$.

Преобразования Пуассона и Фурье сопряжены друг другу:

$$
\left(F_{\lambda, \nu ; \sigma, \varepsilon} f, \varphi\right)_{S}=\left(f, P_{1-n-\lambda, \nu ; \sigma, \varepsilon} \varphi\right)_{\Omega} .
$$

Полюсы преобразования Фурье по $\sigma$, зависящие от $\lambda$, расположены в точках

$$
\sigma=1-n-\lambda-k, \quad \sigma=\lambda+1+l,
$$

где $k, l$ такие же, как и выше. Полюсы (4.6) и (4.9) являются простыми для $\lambda$ общего положения.

\section{§5. Взаимодействие надалгебры с преобразованием Пуассона и Фурье}

В этом параграфе мы указываем в явном виде, как взаимодействуют преобразования Пуассона и Фурье $P_{\lambda, \nu ; \sigma, \varepsilon}$ и $F_{\lambda, \nu ; \sigma, \varepsilon}$ с операторами Ли надгруппы $\widetilde{G}$ в представлении $\widetilde{R}_{\lambda, \nu}$, т. е. с представлением $\widetilde{R}_{\lambda, \nu}$ алгебры Ли $\widetilde{\mathfrak{g}}$ («надалгебры») группы $\widetilde{G}$. Это означает, что мы должны выписать в явном виде композиции $\widetilde{R}_{\lambda, \nu} \circ P_{\lambda, \nu ; \sigma, \varepsilon}(X)$ и $F_{\lambda, \nu ; \sigma, \varepsilon}(X) \circ \widetilde{R}_{\lambda, \nu}$, где $X \in \widetilde{\mathfrak{g}}$. Если $X \in \mathfrak{g}$, то в силу сплетаемости, отмеченной в $\S 4$, ответ прост:

$$
\begin{aligned}
& \widetilde{R}_{\lambda, \nu}(X) \circ P_{\lambda, \nu ; \sigma, \varepsilon}=P_{\lambda, \nu ; \sigma, \varepsilon} \circ T_{2-n-\sigma, \varepsilon}(X), \\
& F_{\lambda, \nu ; \sigma, \varepsilon} \circ \widetilde{R}_{\lambda, \nu}(X)=T_{\sigma, \varepsilon}(X) \circ F_{\lambda, \nu ; \sigma, \varepsilon} .
\end{aligned}
$$

Следовательно, достаточно взять в качестве $X \in \widetilde{\mathfrak{g}}$ элементы из $\widetilde{\mathfrak{g}}$, дополняющие базис в $\mathfrak{g}$ до базиса в $\widetilde{\mathfrak{g}}$. В качестве таких элементов мы возьмем элементы $M_{k}=$ $E_{0 k}+\lambda_{k} E_{k 0}, k=1, \ldots, n$. Как и выше, $E_{i j}, i, j \in\{0,1, \ldots, n\},-$ матричные единицы.

ТЕОРЕма 5.1. Пусть $\sigma$ не является полюсом преобразования Пуассона $P_{\lambda, \nu ; \sigma, \varepsilon}$. Onераторъь $\widetilde{R}_{\lambda, \nu}\left(M_{k}\right), k=1, \ldots, n$, взаимодействуют с этим преобразованием следующим образом:

$$
\widetilde{R}_{\lambda, \nu}\left(M_{k}\right) \circ P_{\lambda, \nu ; \sigma, \varepsilon}=a(\lambda, \sigma) P_{\lambda, \nu ; \sigma+1, \varepsilon+1} \circ K_{\sigma}^{(k)}+b(\lambda, \sigma) P_{\lambda, \nu ; \sigma-1, \varepsilon-1} \circ C^{(k)},
$$

$2 \partial e$

$$
\begin{aligned}
& a(\lambda, \sigma)=\frac{\lambda+\sigma+n-1}{(\sigma+1)(2 \sigma+n-2)}, \quad b(\lambda, \sigma)=\frac{\sigma(\lambda-\sigma+1)}{2 \sigma+n-2}, \\
& K_{\sigma}^{(k)}=-s_{k} \Delta^{(-)}+(2 \sigma+n) X_{k}-(\sigma+p)(\sigma+n-2) s_{k}, \quad k \leqslant p, \\
& K_{\sigma}^{(k)}=s_{k} \Delta^{(-)}+(2 \sigma+n) Y_{k}-(\sigma+q)(\sigma+n-2) s_{k}, \quad k>p, \\
& C^{(k)}=-\delta_{k} s_{k} ;
\end{aligned}
$$

$\Delta^{(-)}, X_{k}, Y_{k}, \delta_{k}$ задаются формулами (2.1), (2.8), (1.1). 
(Отметим, что эта теорема дает следующую реализацию представления $\widetilde{R}_{\lambda, \nu}$ алгебры Ли $\widetilde{\mathfrak{g}}$. Фиксируем $\sigma_{0}$ (общего положения) и $\varepsilon_{0}(=0,1)$. Рассмотрим пространство последовательностей $\varphi=\left\{\varphi_{\sigma}\right\}$, где $\sigma \in \sigma_{0}+\mathbb{Z}, \varepsilon \equiv \varepsilon_{0}+\sigma-$ $\sigma_{0}(\bmod 2)$ и $\varphi_{\sigma} \in \mathscr{D}(S)$. На этом пространстве элементы $X$ из $\mathfrak{g}$ действуют диагонально: $\varphi_{\sigma} \mapsto T_{\sigma, \varepsilon}(X) \varphi_{\sigma}$, а элементы $M_{k} \in \widetilde{\mathfrak{g}}$ переводят $\varphi$ в $\psi$ так, что

$$
\psi_{\sigma}=a(\lambda, \sigma-1) K_{\sigma-1}^{(k)} \varphi_{\sigma-1}+b(\lambda, \sigma+1) C^{(k)} \varphi_{\sigma+1} .
$$

Другие комментарии см. после теоремы 5.2.)

ДокАЗАтЕЛьство. Аналогично формулам (2.6) и (2.7) имеем

$$
\begin{array}{ll}
\widetilde{R}_{\lambda, \nu}\left(M_{k}\right)=-u_{k} \frac{\partial}{\partial u_{0}}+u_{0} \frac{\partial}{\partial u_{k}}, & k \leqslant p, \\
\widetilde{R}_{\lambda, \nu}\left(M_{k}\right)=u_{k} \widetilde{X}_{0}+u_{0} \widetilde{Y}_{k}+(1-n-\lambda) u_{0} u_{k}, & k>p,
\end{array}
$$

где

$$
\widetilde{X}_{0}=\frac{\partial}{\partial u_{0}}-u_{0} \widetilde{D}_{1}, \quad \widetilde{Y}_{k}=\frac{\partial}{\partial u_{k}}-u_{k} \widetilde{D}_{2}, \quad \widetilde{D}_{1}=\sum u_{i} \frac{\partial}{\partial u_{i}}, \quad \widetilde{D}_{2}=\sum u_{j} \frac{\partial}{\partial u_{j}},
$$

переменные $u_{0}$ и $u_{k}$ входят в число локальных координат на $\Omega$, а суммирование в (5.3) происходит по $i=0, \ldots, p$ и по $j=p+1, \ldots, n$, причем какой-нибудь один индекс $i$ и какой-нибудь один индекс $j$ опущены.

Применим оператор $\widetilde{R}_{\lambda, \nu}\left(M_{k}\right)$ к функции (4.4). Для краткости введем обозначение

$$
A=[[u, \stackrel{\circ}{s}]]=-\xi+\eta
$$

где

$$
\xi=u_{1} s_{1}+\cdots+u_{p} s_{p}, \quad \eta=u_{p+1} s_{p+1}+\cdots+u_{n} s_{n} .
$$

Кроме того, для облегчения записи мы опустим индексы $\nu$ и $\varepsilon$ и не будем указывать символ $m$ в обозначении (0.2), так что, например, $A^{\sigma}, A^{\sigma \pm 1}, u_{0}^{\mu}, u_{0}^{\mu+1}$ обозначают соответственно $A^{\sigma, \varepsilon}, A^{\sigma \pm 1, \varepsilon \pm 1}, u_{0}^{\mu, \nu-\varepsilon}, u_{0}^{\mu+1, \nu-\varepsilon+1}$ и т. д.

Мы имеем

$$
\widetilde{R}_{\lambda}\left(M_{k}\right)\left(u_{0}^{1-n-\lambda-\sigma} A^{\sigma}\right)=\delta_{k}\left\{(1-n-\lambda-\sigma) u_{k} u_{0}^{-n-\lambda-\sigma} A^{\sigma}+\sigma u_{0}^{2-n-\lambda-\sigma} A^{\sigma-1}\right\} .
$$

Следовательно,

$$
\widetilde{R}_{\lambda}\left(M_{k}\right) P_{\lambda ; \sigma} \varphi=\delta_{k}\{(1-n-\lambda-\sigma) \cdot \alpha+\sigma \cdot \beta\},
$$

где

$$
\alpha=u_{k} P_{\lambda+1 ; \sigma} \varphi, \quad \beta=P_{\lambda ; \sigma-1}\left(s_{k} \varphi\right) .
$$

$\mathrm{C}$ другой стороны, вычислим преобразование Пуассона $P_{\lambda ; \sigma+1}$ от функции $s_{k} \Delta^{(-)} \varphi$.

Поскольку оператор $\Delta^{(-)}$симметричен относительно формы $(2.3)$, нам надо вычислить $\Delta^{(-)}\left(s_{k} A^{\sigma+1}\right)$. Сначала полезно вычислить

$\Delta^{(-)} A^{\sigma+1}=-(\sigma+1)(\sigma+q-1) A^{\sigma+1}-(\sigma+1)(2 \sigma+n-2) A^{\sigma} \cdot \xi+\sigma(\sigma+1) u_{0}^{2} A^{\sigma-1}$.

Используя это равенство, получаем для $k \leqslant p$

$$
\begin{gathered}
\Delta^{(-)}\left(s_{k} A^{\sigma+1}\right)=(\sigma+2)(\sigma+p) A^{\sigma+1} \cdot s_{k}-(\sigma+1)(2 \sigma+n) A^{\sigma} \cdot \eta \cdot s_{k} \\
+(2 \sigma+2) u_{k} A^{\sigma}+\sigma(\sigma+1) u_{0}^{2} A^{\sigma-1} \cdot s_{k},
\end{gathered}
$$


а для $k>p$

$$
\begin{aligned}
\Delta^{(-)}\left(s_{k} A^{\sigma+1}\right)=- & (\sigma+2)(\sigma+q) A^{\sigma+1} \cdot s_{k}-(\sigma+1)(2 \sigma+n) A^{\sigma} \cdot \xi \cdot s_{k} \\
& +(2 \sigma+2) u_{k} A^{\sigma}+\sigma(\sigma+1) u_{0}^{2} A^{\sigma-1} \cdot s_{k} .
\end{aligned}
$$

Следовательно, для $k \leqslant p$

$P_{\lambda ; \sigma+1}\left(s_{k} \Delta^{(-)} \varphi\right)=-\delta_{k}(\sigma+2)(\sigma+p) \cdot \gamma-(\sigma+1)(2 \sigma+n) \cdot \zeta+(2 \sigma+2) \cdot \alpha+\sigma(\sigma+1) \cdot \beta$,

где

$$
\begin{aligned}
& \gamma=P_{\lambda ; \sigma+1}\left(s_{k} \varphi\right), \\
& \zeta=u_{0}^{-n-\lambda-\sigma} \int_{S} A^{\sigma} \cdot \eta \cdot s_{k} \varphi(s) d s, \quad k \leqslant p, \\
& \zeta=u_{0}^{-n-\lambda-\sigma} \int_{S} A^{\sigma} \cdot \xi \cdot s_{k} \varphi(s) d s, \quad k>p,
\end{aligned}
$$

и та же формула справедлива с заменой $p$ на $q$ для $k>p$.

Теперь вычислим преобразование Пуассона $P_{\lambda ; \sigma+1}$ от функций $X_{k} \varphi$ при $k \leqslant p$ и $Y_{k} \varphi$ при $k>p$. Для этого надо найти соответственно $X_{k}^{*} A^{\sigma+1}$ и $Y_{k}^{*} A^{\sigma+1}$. Используя (2.11), получаем

$$
\begin{aligned}
& X_{k}^{*} A^{\sigma+1}=(\sigma+1) A^{\sigma}\left(u_{k}-\eta s_{k}\right)+(\sigma+p) A^{\sigma+1} \cdot s_{k}, \quad k \leqslant p, \\
& Y_{k}^{*} A^{\sigma+1}=-(\sigma+1) A^{\sigma}\left(u_{k}-\xi s_{k}\right)+(\sigma+q) A^{\sigma+1} \cdot s_{k}, \quad k>p .
\end{aligned}
$$

Следовательно,

$$
\begin{aligned}
P_{\lambda ; \sigma+1}\left(X_{k} \varphi\right) & =(\sigma+1) \cdot \alpha-(\sigma+1) \cdot \zeta+(\sigma+p) \cdot \gamma, & & k \leqslant p, \\
P_{\lambda ; \sigma+1}\left(Y_{k} \varphi\right) & =-(\sigma+1) \cdot \alpha+(\sigma+1) \cdot \zeta+(\sigma+q) \cdot \gamma, & & k>p .
\end{aligned}
$$

Выразим отсюда $(\sigma+1) \cdot \zeta$ и подставим в формулу $(5.5)$ для $k \leqslant p$ и сделаем то же с заменой $p$ на $q$ для $k>p$. Затем выразим $\alpha$ и подставим в (5.4). После приведения подобных членов получим (5.1).

Из формул (2.11)-(2.13) следует, что сопряженным относительно (2.3) оператором к $K_{\sigma}^{(k)}$ является $K_{1-n-\sigma}^{(k)}$. В силу условия сопряженности (4.8) мы получаем следующий результат.

Теорема 5.2. Пусть $\sigma$ не является полюсом преобразования Фурье $F_{\lambda, \nu ; \sigma, \varepsilon}$. Операторь $\widetilde{R}_{\lambda, \nu}\left(M_{k}\right)$ взаимодействуют с этим представлением следующим образом:

$$
\begin{aligned}
F_{\lambda, \nu ; \sigma, \varepsilon} \circ \widetilde{R}_{\lambda, \nu}\left(M_{k}\right)=- & a(1-n-\lambda, \sigma) K_{1-n-\sigma}^{(k)} \circ F_{\lambda, \nu ; \sigma+1, \varepsilon+1} \\
& -b(1-n-\lambda, \sigma) C^{(k)} \circ F_{\lambda, \nu ; \sigma-1, \varepsilon-1} .
\end{aligned}
$$

Обсудим формулы (5.1) и (5.6) и структуру коэффициентов $a, b$ для $\lambda$ общего положения $(2 \lambda \notin \mathbb{Z})$. Мы видим, что для $\sigma$ общего положения последовательность $\sigma+\mathbb{Z}$ есть своего рода «пространство представления» для представления $\widetilde{R}_{\lambda, \nu}$ алгебры Ли $\widetilde{\mathfrak{g}}$.

Коэффициенты (5.2) содержат множители

$$
\beta_{1}(\lambda, \sigma)=\sigma+\lambda+n-1, \quad \beta_{2}(\lambda, \sigma)=\sigma-\lambda-1,
$$


являющиеся линейными функциями от $\sigma$, зависящими от $\lambda$. В (4.5) показатели множителей перед рядами - это как раз $-\beta_{1}$ и $\beta_{2}$.

$\mathrm{C}$ другой стороны, эти линейные функции должны запрещать попадание в полюсы преобразования Пуассона, см. формулы (4.6). Следовательно, функция $\beta_{1}$ должна обращаться в нуль в точке $\sigma=1-n-\lambda$ (один шаг влево от самой левой точки во второй из последовательностей (4.6)), а функция $\beta_{2}$ должна обращаться в нуль в точке $\sigma=\lambda+1$ (один шаг вправо от самой правой точки в первой из последовательностей (4.6)). Это объясняет появление множителей (5.7) в формуле (5.1). Функции $\beta_{i}$ - это барьерные функиии. С другой стороны, нули этих функций являются крайними полюсами преобразования Фурье, см. (4.9). Следовательно, каждая из двух последовательностей (4.9) есть своего рода «инвариантное подпространство» для $\widetilde{R}_{\lambda, \nu}$. Такое подпространство в самом деле существует: это пространство струй, на котором действует представление $M_{\lambda, \nu}$, см. $\S 3$. Как уже было сказано, для $\lambda$ общего положения это представление диагонализуемо.

Аналогично (двойственным образом) дело обстоит с коэффициентами $a, b$ в соотношении (5.6). Здесь появляются функции

$$
\beta_{1}(1-n-\lambda, \sigma)=\sigma-\lambda, \quad \beta_{2}(1-n-\lambda, \sigma)=\sigma+\lambda+n-2 .
$$

Они должны запрещать попадание в полюсы преобразования Фурье (см. (4.9)) и, следовательно, имеют указанный вид. Их нули - крайние полюсы преобразования Пуассона. Следовательно, каждая из последовательностей (4.6) есть «инвариантное подпространство». Такое пространство в самом деле существует: это пространство $\Sigma_{\nu}(\Omega)$ обобщенных функций, сосредоточенных на $S$, где действует представление $L_{\lambda, \nu}$, cм. $\S 3$. Для $\lambda$ общего положения оно диагонализуемо.

\section{§6. Случаи $p=1$ и $q=1$}

Случаи $p=1$ (тогда $q=n-1$ ) и $q=1$ (тогда $p=n-1$ ) имеют некоторые отличия от общего случая $p>1, q>1$. Для нашей задачи основное отличие заключается в том, что группа $K$ имеет нетривиальный (одномерный) централизатор в $\widetilde{\mathfrak{g}}$. Он порожден элементом $M_{1}$ для $p=1$ и $M_{n}$ для $q=1$. Это значительно облегчает вычисления в теореме 5.1.

Сейчас эту теорему удобно доказывать так. Например, пусть $p=1$. Поскольку $M_{1}$ коммутирует с $\mathfrak{k}$, операторы $K_{\sigma}^{(1)}$ и $C^{(1)}$ коммутируют с вращениями из группы $K$, и их вычисление оказывается значительно проще, чем вычисление операторов в $\S 5$. Затем используем соотношение коммутации $\left[M_{1}, L_{1 k}\right]=M_{k}$. Оно дает

$$
\widetilde{R}_{\lambda, \nu}\left(M_{k}\right)=\left[\widetilde{R}_{\lambda, \nu}\left(M_{1}\right), R_{\lambda, \nu}\left(L_{1 k}\right)\right],
$$

и остается вычислить $K_{\sigma}^{(k)}$ и $C^{(k)}$ по формулам

$$
\begin{aligned}
& K_{\sigma}^{(k)}=K_{\sigma}^{(1)} T_{2-n-\sigma}\left(L_{1 k}\right)-T_{1-n-\sigma}\left(L_{1 k}\right) K_{\sigma}^{(1)}, \\
& C^{(k)}=C^{(1)} T_{2-n-\sigma}\left(L_{1 k}\right)-T_{3-n-\sigma}\left(L_{1 k}\right) C^{(1)} .
\end{aligned}
$$

Такой способ годится и в общем случае $p>1, q>1$, однако количество вычислений оказывается не меньше, чем в доказательстве теоремы 5.1 в $\S 5$.

Для случаев $p=1$ и $q=1$ многообразие $\mathscr{X}$ есть соответственно однополостный гиперболоид и одна пола двуполостного гиперболоида (во втором случае 
$\mathscr{X}$ есть пространство Лобачевского размерности $n-1$, это - риманово симметрическое пространство). Многообразие $S$ есть сечение конуса $\{x:[x, x]=0\}$ гиперплоскостью $x_{1}=1$ для $p=1$ и $x_{n}=1$ для $q=1$. Оно есть сфера $S_{2}$ для $p=1$ и сфера $S_{1}$ для $q=1$. Пусть $\Delta_{S}$ обозначает оператор Лапласа-Бельтрами на $S$. Он есть $\Delta_{2}$ для $p=1$ и $\Delta_{1}$ для $q=1$, так что $\Delta^{(-)}=\Delta_{S}$ для $p=1$ и $\Delta^{(-)}=-\Delta_{S}$ для $q=1$. Представления группы $G$, связанные с конусом, нумеруются с помощью $\sigma \in \mathbb{C}\left(\right.$ без $\varepsilon$ ). Представление $T_{\sigma}$ действует в $\mathscr{D}(S)$ по формуле $(2.2)$, где надо заменить $|s g|$ на $(s g)_{1}$ при $p=1$ и на $(s g)_{n}$ при $q=1$.

При $p=1$ пространство $H$-инвариантов в $\mathscr{D}^{\prime}(S)$ в представлении $T_{\sigma}$ двумерно (за исключением случая $n=3, \sigma=-1,-2, \ldots$, когда оно трехмерно), базис образован обобщенными функциями $\theta_{\sigma, \varepsilon}, \varepsilon=0,1$, см. $(4.1)$, или их вычетами. Для теорем 5.1 и 5.2 надо сделать следующие изменения:

$$
\begin{aligned}
& K_{\sigma}^{(1)}=-\Delta_{S}-(\sigma+1)(\sigma+n-2), \\
& K_{\sigma}^{(k)}=s_{k} \Delta_{S}+(2 \sigma+n) Y_{k}-(\sigma+n-1)(\sigma+n-2) s_{k}, \quad k \geqslant 2, \\
& C^{(1)}=1, \quad C^{(k)}=-s_{k}, \quad k \geqslant 2 .
\end{aligned}
$$

При $q=1$ группа $H$ совпадает с $K$. Пространство $K$-инвариантов в $\mathscr{D}(S)$ в представлении $T_{\sigma}$ состоит из констант. Представления группы $\widetilde{G}=S O_{0}(n, 1)$, связанные с конусом, нумеруются с помощью $\lambda \in \mathbb{C}$ (без $\nu$ ). Они действуют в однородных функциях степени однородности $\lambda$ на поле конуса $\mathscr{C}^{+}=\{x$ : $\left.[x, x]=0, x_{n}>0\right\}$. Многообразие $\Omega$ есть сфера - сечение этого конуса гиперплоскостью $u_{n}=1$. В $\mathscr{D}(\Omega)$ представления $\widetilde{R}_{\lambda}$ действуют по формуле

$$
\left(\widetilde{R}_{\lambda}(g) f\right)(u)=f\left(u g /(u g)_{n}\right)(u g)_{n}^{1-n-\lambda} .
$$

Их ограничения на $G$ - это канонические представления $R_{\lambda}$ группы $G$.

Представления Пуассона и Фурье, кроме $\lambda$ и $\sigma$, зависят еще от параметра $\nu=0,1$. Мы определяем преобразование Пуассона $P_{\lambda, \nu, \sigma}: \mathscr{D}(S) \rightarrow \mathscr{D}(\Omega)$ и преобразование $Ф$ урье $F_{\lambda, \nu, \sigma}: \mathscr{D}(\Omega) \rightarrow \mathscr{D}(S)$ формулами $(4.4)$ и $(4.7)$, в которых надо опустить $\varepsilon$. Заметим, что $[[u, \stackrel{\circ}{s}]] \geqslant 0$ для всех $u \in \Omega, s \in S$. Функции из образа преобразования Пуассона имеют четность $\nu$ по $u_{0}$, а преобразование Фурье обращается в нуль на функциях противоположной четности. Для теорем 5.1 и 5.2 надо сделать следующие изменения:

$$
\begin{gathered}
K_{\sigma}^{(k)}=s_{k} \Delta_{S}+(2 \sigma+n) X_{k}-(\sigma+n-2)(\sigma+n-1) s_{k}, \quad k<n, \\
K_{\sigma}^{(n)}=-\Delta_{S}-(\sigma+1)(\sigma+n-2), \\
C^{(k)}=s_{k}, \quad k<n, \quad C^{(n)}=-1 .
\end{gathered}
$$

В частности, пусть $p=2, q=1$. Тогда представление $\widetilde{R}_{\lambda}$ надгруппы $\widetilde{G}=$ $\mathrm{SO}_{0}(3,1)$ действует сдвигами в пространстве $\mathscr{D}_{-\lambda-2}\left(\mathscr{C}^{+}\right)$функций класса $C^{\infty}$ и однородных степени $-\lambda-2$ на конусе $\mathscr{C}^{+}=\left\{x:-x_{0}^{2}-x_{1}^{2}-x_{2}^{2}+x_{3}^{2}=0, x_{3}>0\right\}$. Сечение $\mathscr{Z}$ этого конуса плоскостью $x_{0}+x_{3}=2$ можно отождествить с $\mathbb{C}:$ точке $z=x+i y$ отвечает точка $\zeta=(1-z \bar{z}, 2 x, 2 y, 1+z \bar{z})$ из $\mathscr{Z}$. С другой стороны, пространство $\mathbb{R}^{4}$ можно реализовать как пространство эрмитовых матриц

$$
x=\frac{1}{2}\left(\begin{array}{cc}
x_{3}-x_{0} & x_{1}-i x_{2} \\
x_{1}+i x_{2} & x_{3}+x_{0}
\end{array}\right) .
$$


Группа $\mathrm{SL}(2, \mathbb{C})$ действует на нем по формуле $x \mapsto \bar{g}^{\prime} x g$. Это дает гомоморфизм группы $\mathrm{SL}(2, \mathbb{C})$ на группу $\mathrm{SO}_{0}(3,1)$ с ядром $\{ \pm E\}$, так что $\widetilde{R}_{\lambda}$ есть представление и группы $\mathrm{SL}(2, \mathbb{C})$. Пространство $\mathscr{D}_{-\lambda-2}\left(\mathscr{C}^{+}\right)$может быть реализовано как некоторое пространство функций на сечении $\mathscr{Z}$. В этой реализации представление $\widetilde{R}_{\lambda}$ группы $\mathrm{SL}(2, \mathbb{C})$ дается в точности формулой $(0.1)$, так что наша конструкция канонических представлений в случае $p=2, q=1$ дает канонические представления из [2] (надо еще взять ограничение на круг $D$ ).

\section{ЛитеРАТУРА}

1. Березин $\Phi$. А. Квантование в комплексных симметрических пространствах. Изв. AH CCCP, сер. мат., 39, № 2, 363-402 (1975).

2. Вершик А. М., Гельфанд И. М., Граев М. И. Представления группы $\operatorname{SL}(2, R)$, где $R$ - кольцо функций. УМН, 28, вып. 5, 83-128 (1973).

3. Молчанов B. Ф. Представления псевдоортогональной группы, связанные с конусом. Матем. сб., 81, №3, 358-375 (1970).

4. Молчанов B. Ф. Сферические функции на гиперболоидах. Матем. сб., 99, No. 2, 139-161 (1976).

5. Молчанов В. Ф. Формула Планшереля для гиперболоидов. Труды МИАН, 147, 65-85 (1980).

6. Молчанов В. Ф. Гармонический анализ на однородных пространствах. В кн.: Итоги науки и техники. Современные проблемы математики. Фундаментальные направления, т. 59, ВИНИТИ, М., 1990, с. 5-144.

7. Molchanov V. F. Quantization on para-Hermitian symmetric spaces. In: Contemporary Mathematical Physics. Amer. Math. Soc. Transl. Ser. 2 (Adv. Math. Sci., Vol. 31), Vol. 175, 1996, pp. 81-95.

8. Molchanov V. F., Volotova N. B. Finite dimensional analysis and polynomial quantization on a hyperboloid of one sheet. In: Proc. Tambov Summer School-Seminar "Harmonic analysis on homogeneous spaces" (Aug. 26-31, 1996). Вестник Тамбовского унив., 3, вып. 1, 65-78 (1998).

9. Molchanov V. F. Canonical representations and overgroups. Preprint Math. Inst. Univ. Leiden, No. MI 2002-05 (2002).

10. Molchanov V. F. Canonical representations and overgroups. In: Lie Groups and Symmetric Spaces. Amer. Math. Soc. Transl. Ser. 2 (Adv. Math. Sci., Vol. 54), Vol. 210, 2003, pp. 213-224.

11. Molchanov V. F. Canonical representations and overgroups for hyperboloids of one sheet and Lobachevsky spaces. Acta Appl. Math., 86, No. 1, 115-129 (2005).

12. Неретин Ю. А. Действие надалгебры в планшерелевском разложении и операторы сдвига в мнимом направлении. Изв. РАН, сер. матем., 66, №5, 171-182 (2002).

Тамбовский государственный университет email: molchano@molchano.tstu.ru
Поступило в редакцию 25 ноября 2003 г. 